\title{
Ergogenic Value of Oxygen Supplementation in Patients with Idiopathic Pulmonary Fibrosis with Isolated Exertional Oxygen Desaturation
}

\author{
loannis Vogiatzis ${ }^{a} \quad$ Demosthenes Bouros ${ }^{b}$ \\ aDepartment of Sport, Exercise and Rehabilitation, Northumbria University Newcastle, Newcastle upon Tyne, UK; ${ }^{\text {b}} 1$ st \\ Department of Respiratory Medicine, National and Kapodistrian University of Athens Medical Centre, Athens, Greece
}

Idiopathic pulmonary fibrosis (IPF) is a progressive diffuse parenchymal disease with a poor prognosis [1-3]. Progression of IPF is reflected by a decline in lung function, worsening of dyspnea and exercise capacity, and deterioration in health-related quality of life $[4,5]$ Optimal management of IPF requires a multidimensional approach, including both pharmacological therapy and supportive care. Pulmonary hypertension often complicates the course of IPF and may even be found in patients with preserved lung function. Pulmonary hypertension in IPF patients is associated with decreased exercise capacity and a worse prognosis.

The underlying physiological mechanisms regulating the effectiveness of oxygen supplementation during exercise in patients with IPF with isolated exertional oxygen desaturation are largely unknown. The elegant study by Dipla and colleagues [6] examined the effects of oxygen supplementation during sub-maximal exercise (vs. medical air) on cerebral and locomotor local muscle oxygen availability, and systemic hemodynamics.

In this prospective, randomized, cross-over, placebocontrolled study, 13 IPF patients without resting hypoxemia, but a significant arterial oxygen desaturation during maximal cardiopulmonary exercise testing, underwent 2 steady-state exercise trials on the cycle ergometer (equivalent to $65 \%$ peak-workload), breathing either oxygen-enriched air $\left(\mathrm{FiO}_{2}: 40 \%\right)$ or medical air (sham oxygen; $\mathrm{FiO}_{2}: 21 \%$ ). Cerebral/skeletal muscle oxygenation was monitored (by near-infrared-spectroscopy) and beat-by-beat hemodynamics by photoplethysmography. The study concluded that correcting exercise-induced arterial oxygen desaturation with oxygen supplementation, enhanced exercise tolerance, prevented the decline in cerebral oxygen availability, and improved local muscle oxygen availability, whilst lessened dyspnea and leg discomfort, thus suggesting an efficacy of acute oxygen supplementation during exercise in IPF patients.

Discussion of why the oxygen compared to the medical air protocol significantly increased endurance time ( $15.2 \pm 5.0$ vs. $10.0 \pm 4.3 \mathrm{~min}$, respectively) during exercise with same average exercise intensity in each protocol is of great interest to elucidate the physiological mechanisms enhancing exercise tolerance in IPF. The logic is as follows:

1. Suppose that there was one dominant limiting factor to endurance time to consider among the physiological (cardiac output, cerebral oxygen availability, peripheral local muscle oxygen availability) and perceptional (dyspnea and leg discomfort) variables recorded.

2. At the time point marking the limit of tolerance in the air protocol (i.e., isotime: when work completed was the same in air and oxygen protocols), one would expect that the value of the dominating limiting factor would be less altered (from resting values) in the oxygen protocol than in the air protocol. According to the results of the study, the candidate variables were: (i) the lower dyspnea, (ii) the lower leg discomfort, (iii) 
the lower locomotor local muscle de-oxygenation (i.e., greater locomotor local muscle oxygen availability), and (iv) the lower cerebral de-oxygenation (i.e., greater cerebral oxygen availability). Cardiac output does not fit this pattern because there were not any differences between the air and oxygen protocols, so circulatory limitation was not a limiting factor.

3. At the time point marking limit of tolerance in the oxygen protocol, one would expect the value of the dominating limiting factor to have reached the same alteration (from resting values) as at the limit of tolerance in the air protocol. Dyspnea and cerebral de-oxygenation are not candidates because they were still lower at the limit of tolerance in the oxygen protocol compared to the limit of tolerance in the air protocol. The logic is that if dyspnea or cerebral oxygen availability limited endurance time, then endurance should have been even longer in the oxygen protocol than it was because the perturbations had not yet reached the value seen in the air protocol.

4. The only variables examined that fit the pattern expected for a limiting factor was locomotor local muscle de-oxygenation (local muscle oxygen availability) and leg discomfort. In fact, at exercise termination perceived leg discomfort and locomotor local muscle deoxygenation were not significantly different between the air and oxygen protocols.

5. These data and the above analysis then suggest that the reason for reaching a limitation in each protocol was having reached the same degree of locomotor muscle de-oxygenation and leg discomfort, which took longer in the oxygen than in the air protocol. These findings, in turn, highlight the value of oxygen supplementation during exercise training as part of pulmonary rehabilitation in patients with IPF. Greater locomotor muscle oxygen availability would delay metabolic acidosis and attenuate locomotor muscle fatigue, thereby increasing the time that IPF patients can exercise during a session of pulmonary rehabilitation. This would, in turn, be expected to augment the benefits of pulmonary rehabilitation in improving functional capacity and autonomy in this population.

Of interest is that the duration of exercise with oxygen supplementation (on average $15 \mathrm{~min}$ ) is still quite limited to optimize the physiological adaptations of exercise training in this population. Considering that oxygen supplementation is not always available in the hospital- and/ or the community-based pulmonary rehabilitation setting, alternative modalities of exercise training, such as intermittent exercise, may be considered in patients with IPF with isolated exertional hypoxemia. Intermittent exercise training, as part of pulmonary rehabilitation, has been shown to be feasible and efficacious in IPF patients in improving exercise tolerance, functional capacity, pulmonary function, dyspnea and health-related quality of life, thereby suggesting that this training modality may be considered the standard care for IPF [7]. A recently published review article introduces the principles and different modalities of exercise training and summarizes the effects of various types of exercise training in patients with interstitial lung diseases, including IPF [8].

\section{Conflict of Interest Statement}

We declare we have no conflicts of interest.

\section{Funding Sources}

No funding was received.

\section{References}

1 Bouros D, Tzilas V, Tzouvelekis A. Diagnostic guidelines for IPF: when art meets science. Lancet Respir Med. 2018;6(11):812-4.

2 Pitsiou G, Papakosta D, Bouros D. Pulmonary hypertension in idiopathic pulmonary fibrosis: a review. Respiration. 2011;82(3):294304.

3 Tzouvelekis A, Tzilas V, Papiris S, Aidinis V, Bouros D. Diagnostic and prognostic challenges in idiopathic pulmonary fibrosis: a patient's "Q and A" approach. Pulm Pharmacol Ther. 2017;42:21-4.
4 Papadopoulos CE, Pitsiou G, Karamitsos TD, Karvounis HI, Kontakiotis T, Giannakoulas $\mathrm{G}$, et al. Left ventricular diastolic dysfunction in idiopathic pulmonary fibrosis: a tissue Doppler echocardiographic study. Eur Respir J. 2008;31:701-6.

5 Wuyts WA, Wijsenbeek M, Bondue B, Bouros D, Bresser P, Robalo Cordeiro C, et al. Idiopathic pulmonary fibrosis: best practice in monitoring and managing a relentless fibrotic disease. Respiration. 2020;99(1):73-82.
6 Dipla K, Boutou AK, Markopoulou K, Pitsiou G, Papadopoulos S, Chatzikosti A, et al. Exertional desaturation in idiopathic pulmonary fibrosis: the role of oxygen supplementation in modifying cerebral-skeletal muscle oxygenation and systemic hemodynamics. Respiration. 2021.

7 Vainshelboim B, Oliveira J, Yehoshua L, Weiss I, Fox BD, Fruchter O, et al. Exercise trainingbased pulmonary rehabilitation program is clinically beneficial for idiopathic pulmonary fibrosis. Respiration. 2014;88(5):378-88.

8 Armstrong M, Vogiatzis I. Personalized exercise training in chronic lung diseases. Respirology. 2019;24(9):854-62. 